\title{
Azimuthal particle correlations as a probe of collectivity in deep inelastic ep collisions at HERA
}

\section{Abt* for the ZEUS collaboration} Max-Plank-Institut for Physics

E-mail: isa@mpp.mpg.de

\begin{abstract}
Measurements of two-particle azimuthal correlations, $c_{n}\{2\}$, where $n=1-4$ denotes the harmonics, are presented for neutral current deep-inelastic ep scattering events, collected with the photon virtuality, $Q^{2}$, above $5 \mathrm{GeV}^{2}$ with the ZEUS detector at HERA at a centre-of-mass energy, $s$, of $\sqrt{s}=318 \mathrm{GeV}$. The $c_{n}\{2\}$ were determined for charged particles with laboratory pseudo-rapidities, $\eta$, in the range $-1.5<\eta<2$ and transverse momenta, $p_{\mathrm{T}}$, in the range $0.1<p_{\mathrm{T}}<5.0 \mathrm{GeV} / \mathrm{c}$. Distributions are presented as functions of the event multiplicity, $N_{c h}$, the separations in pseudorapidity, $\Delta \eta$, and the separation in $p_{\mathrm{T}}, \Delta p_{\mathrm{T}}$. The data do not provide evidence for any collective effects.
\end{abstract}

XXVII International Workshop on Deep-Inelastic Scattering and Related Subjects - DIS2019

8-12 April, 2019

Torino, Italy

${ }^{*}$ Speaker. 


\section{Experimental setup and Monte Carlo}

Collective effects indicating the emergence of many-body systems in local thermal equilibrium were first observed in heavy-ion collisons and later also in smaller systems, even as small as $p p$ collisions. The analysis presented here represents a search for possible collective effects in the $e p$ system.

The data were taken with the ZEUS [1] detector at HERA from 2003 to 2007. During this period, the HERA accelerator collided $27.5 \mathrm{GeV}$ electrons or positrons ${ }^{1}$ and $920 \mathrm{GeV}$ protons, resulting in a centre-of-mass energy of $\sqrt{s}=318 \mathrm{GeV}$. The rapidity of the centre-of-mass frame differs by 5.8 units from the laboratory frame, following the direction of the proton beam. The data correspond to an integrated luminosity of $366 \pm 6 \mathrm{pb}^{-1}$.

A total of 45 million NC events were selected using standard ZEUS selection criteria [2, 3]. Charged particles were selected using standard ZEUS tracking selection criteria and the requirement that they could be assigned to the primary vertex. Physical primary particles were defined as charged particles with a mean proper lifetime, $\tau>1 \mathrm{~cm} / c$, which were produced directly or from the decay of a particle with $\tau<1 \mathrm{~cm} / c$. The scattered electron candidate used to identify the NC DIS event was not included in the analysis of correlations. The transverse momentum, $p_{\mathrm{T}}$, and laboratory pseudo-rapidity, $\eta$, intervals selected for the analysis were $0.1<p_{\mathrm{T}}<5.0 \mathrm{GeV} / \mathrm{c}$ and $-1.5<\eta<2.0$, respectively.

The LEPTO 6.5 [4] and ARIADNE 4.12 [5] Monte Carlo event generators were used to simulate events coming from standard physics mechanisms. Both generators were interfaced to PYTHIA 5.724 and JETSET 7.410 [6] to handle the initial hard scattering, hadronisation, and decays. The correlations determined for these MC events were compared to the correlations as observed in the data. The MC events were also used to determine efficiencies and associated systematic uncertainties.

The two generators differ mostly in their treatment of the quantum chromo-dynamics (QCD) cascade process. In LEPTO, the cascade is treated with a DGLAP-based backward evolution shower. ARIADNE treats the cascade within the colour dipole model (CDM). Both the initialstate radiation (before virtual photon exchange) and final state radiation are treated independently in LEPTO while in ARIADNE they are treated on equal footing as final state emissions.

Figure 1 shows the multiplicity and $\eta$ distributions as measured and predicted from the LEPTO and ARIADNE generators, respectively. The overall description of the data is good. Due to the moving centre-of-mass frame many particles escape through the beam-pipe; especially most of the proton-remnant does.

\section{Azimuthal correlations}

The search for collectivity uses particle correlations in the transverse plane to probe the dynamics of the produced medium. Final state anisotropies along any common plane of symmetry $\left(\Psi_{m}\right)$ in the initial state reveal the emergence of a collectivity. The two-particle correlation coeffi-

\footnotetext{
${ }^{1}$ Hereafter, "electron" refers to both electrons and positrons unless otherwise stated.
} 

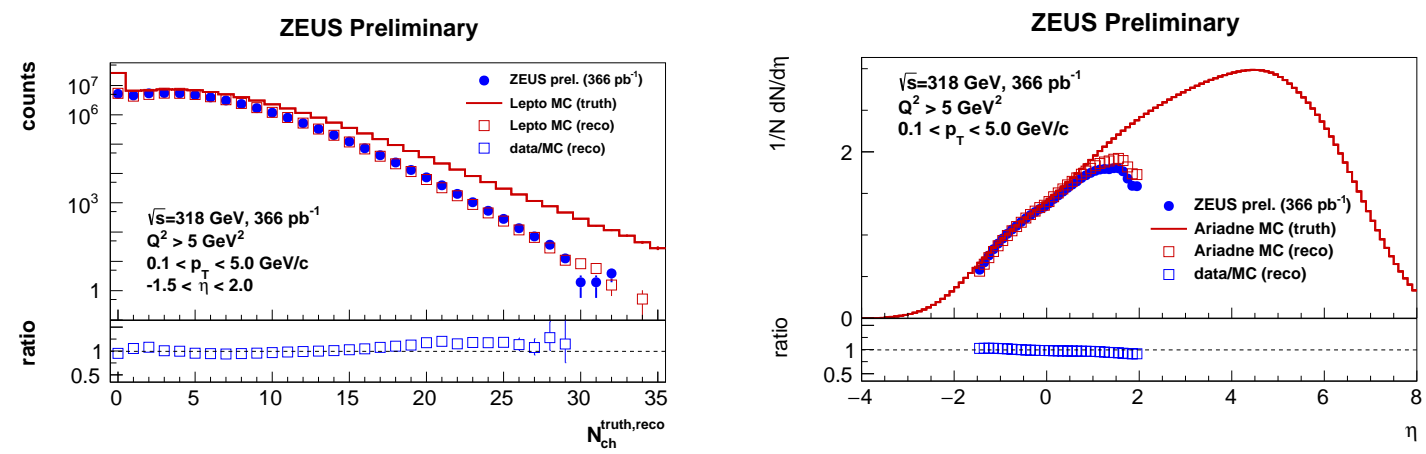

Figure 1: Uncorrected distributions of multiplicity (left) and pseudo-rapidity $\eta$ for $N_{\mathrm{ch}} \geq 5$ (right) in data compared to MC simulations using the Lepto and Ariadne generators, respectively. The reconstructed Monte Carlo multiplicity distribution was normalised to that in data using the total number of events with $N_{\mathrm{ch}}>0$. Generator level distributions were normalised by the same factor. The statistical uncertainties are smaller than the marker size.

cients reported in this paper are defined as

$$
c_{n}\{2\}=\sum_{e}^{N}\left[\sum_{i, j>i}^{M} w_{i} w_{j} \cos \left[n\left(\varphi_{i}-\varphi_{j}\right)\right]\right]_{e} / \sum_{e}^{N}\left[N_{\mathrm{ch}}\left(N_{\mathrm{ch}}-1\right)\right]_{e},
$$

where $n$ indicates the harmonics, the first sum runs over all events, $N$, and the sums running over $i$ and $j$ run over all selected charged particles in the event which has the multiplicity $M$. The tracking efficiency corrections enter the analysis in the form of a pair of weights, $w_{i} w_{j}$. These weights approximately do not affect the ratio described in Eq. (2.1) because the true number of charged primary particles, $N_{\mathrm{ch}}$, in a given event was estimated using the weighted sum running over the $M$ reconstructed tracks:

$$
N_{\mathrm{ch}}=\sum_{i}^{M} w_{i}
$$

\section{Results}

The two-particle correlation coefficients as defined in Eq. (2.1) for the harmonics $n=1-4$ are shown in Fig. 2. Without the requirement of a rapidity separation, all $c_{n}\{2\}$ peak at low multiplicity for all $n$. This feature is strongly reduced for $|\Delta \eta|>2$, indicating that the $c_{n}\{2\}$ at small multiplicities are dominated by short-range correlations. The correlation coefficients $c_{1}\{2\}$ and $c_{3}\{2\}$ become negative for large $|\Delta \eta|$, which is expected from the effects of momentum conservation, e.g. when back-to-back di-jet processes dominate particle production. Both $c_{2}\{2\}$ and $c_{4}\{2\}$ are consistent with zero within the systematic uncertainties for the requirement of a $|\Delta \eta|>2$ separation.

The predictions based on the LEPTO and ARIADNE generators are compared to the measured correlation coefficients $c_{1}\{2\}$ and $c_{2}\{2\}$ as a function of $N_{c h}$ in Fig. 3. The basic features are described by both models. However, $c_{1}\{2\}$ is better described by the ARIADNE based predictions while $c_{2}\{2\}$ is better described by the LEPTO based MC. The predictions for $c_{2}\{2\}$ as functions of $\Delta \eta$ and $\Delta p_{\mathrm{T}}$ are compared to data in Fig. 4. The predictions based on LEPTO describe the 

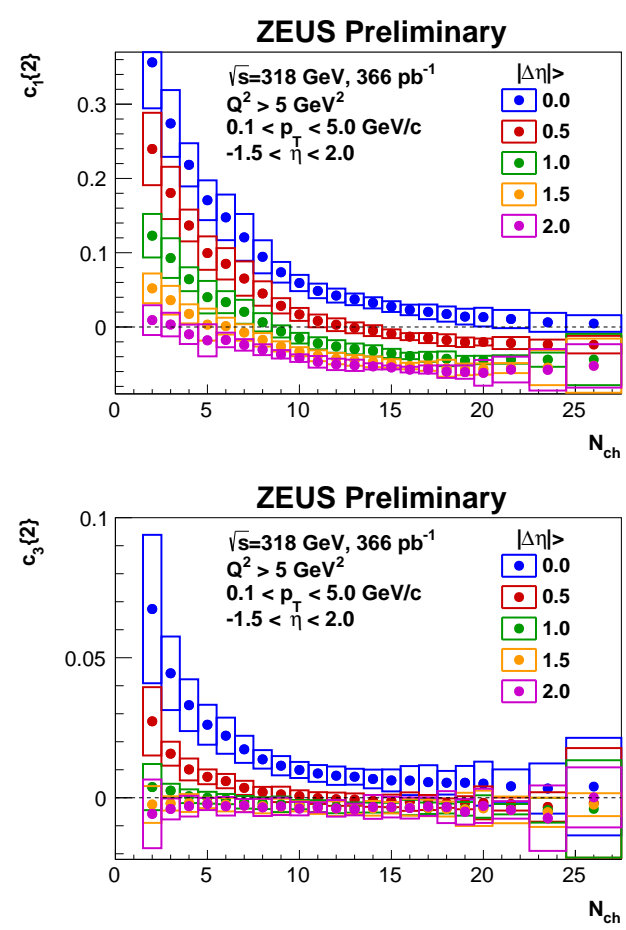

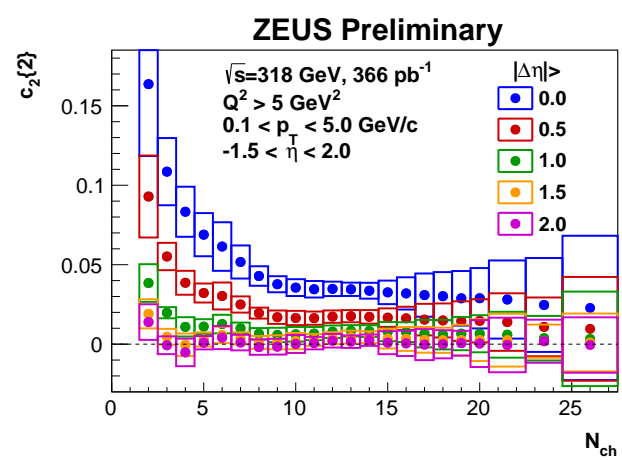

ZEUS Preliminary

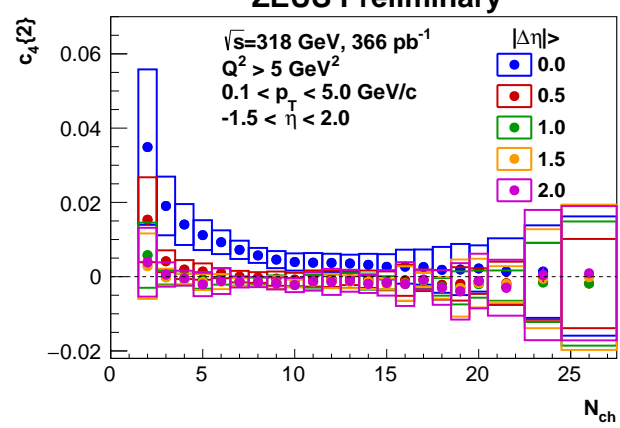

Figure 2: The correlation coefficients $c_{n}\{2\}, n=1,2,3,4$, as a function of $N_{\text {ch }}$ for different rapidity separations. The statistical uncertainties are shown as vertical lines although they are typically smaller than the marker size. Systematic uncertainties are shown as boxes.

data quite well; the predictions based on ARIADNE describe the basic features. In Fig. 5, MC predictions for $c_{1}\{2\}$ and $c_{2}\{2\}$ as a function of $\left\langle p_{\mathrm{T}}\right\rangle=\left(p_{\mathrm{T}, 1}+p_{\mathrm{T}, 2}\right) / 2$ are compared to data. The correlation coefficient $c_{1}\{2\}$ is better described by the ARIADNE based predictions; both MC predictions describe the basic features of the measured $c_{2}\{2\}$ correlation coefficients.

\section{Summary and Outlook}

The two-particle coefficients $c_{n}\{2\}$ were presented as functions $N_{c h}$ for the harmonics $n=1-4$. The correlations coefficients $c_{1}\{2\}$ and $c_{3}\{2\}$ become negative for large $|\Delta \eta|$, reflecting the effects of momentum conservation. The coefficients $c_{2}\{2\}$ and $c_{4}\{2\}$ are compatible with zero for large $|\Delta \eta|$. Collective effects would be expected to cause non-zero $c_{2}\{2\}$ and $c_{4}\{2\}$.

Predictions based on the MC event generators LEPTO and ARIADNE were compared to the measured correlation coefficients as functions of $\Delta \eta, \Delta p_{\mathrm{T}}$ and $\left\langle p_{\mathrm{T}}\right\rangle$. Both models are not able to describe all details of all measured distributions. However, both the LEPTO and ARIADNE based predictions describe the general features of the $c_{n}\{2\}$ distributions reasonably well. In summary, the analysis does not provide evidence for collectivity in $\mathrm{NC}$ ep deep inelastic scattering.

In the colour-dipole picture of $e p$ scattering, the photon fluctuates into a hadronic system. The lower $Q^{2}$, the more time is available for the system to grow. It is planned to extend the analysis to photoproduction events to investigate this $\gamma p$ system. 

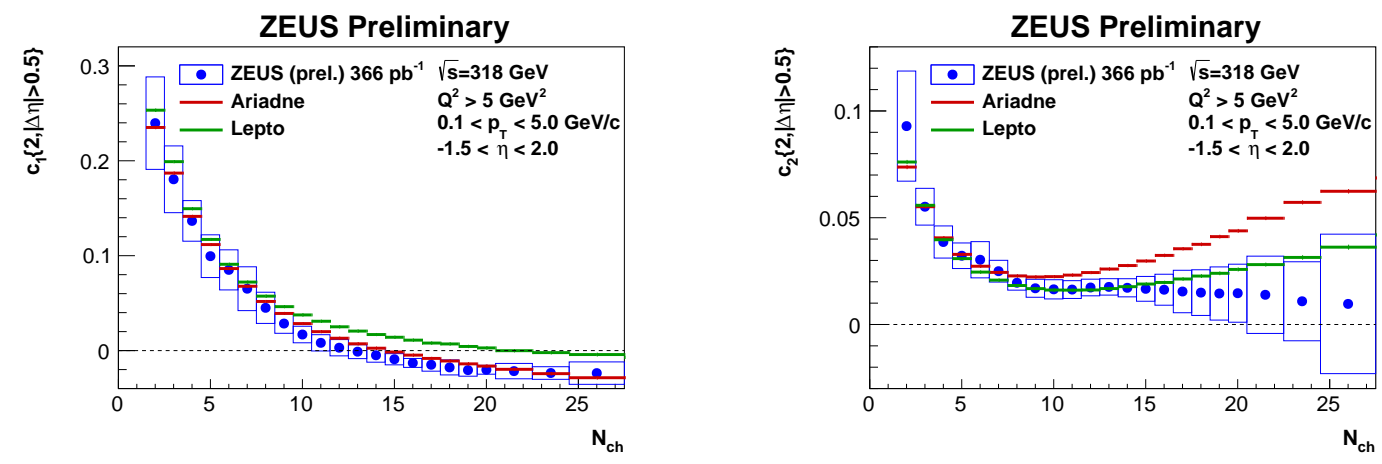

Figure 3: The correlation coefficients $c_{n}\{2\}, n=1,2$, as a function of $N_{\mathrm{ch}}$ for $|\Delta \eta|>0.5$. The statistical uncertainties are shown as vertical lines although they are typically smaller than the marker size. Systematic uncertainties are shown as boxes. Also shown are predictions based on the LEPTO and ARIADNE MC generators.
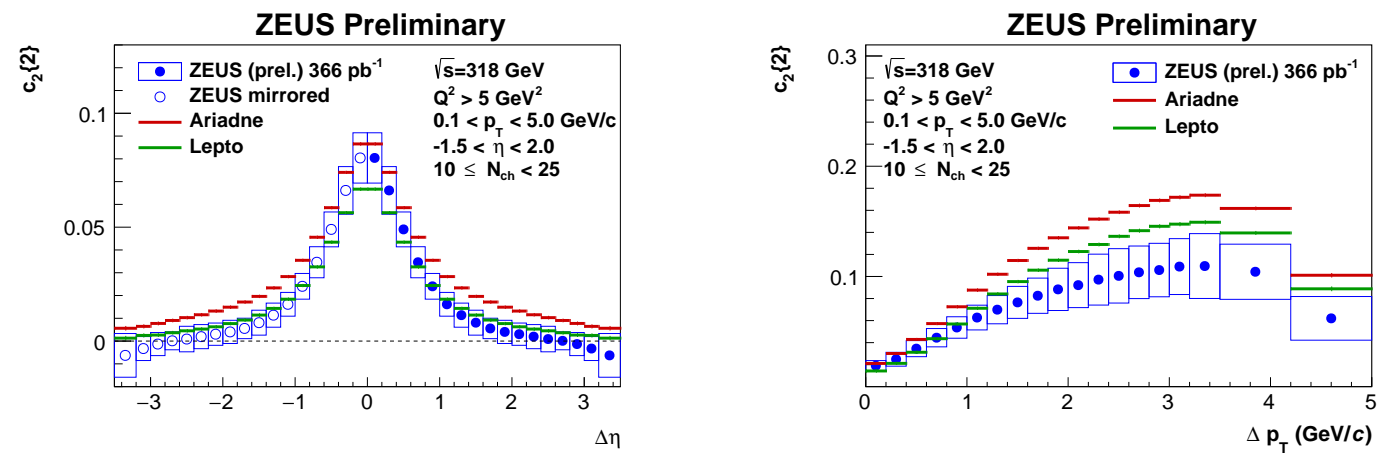

Figure 4: The correlation coefficient $c_{2}\{2\}$ as functions of $\Delta \eta$ and $\Delta p_{\mathrm{T}}$. The statistical uncertainties are shown as vertical lines although they are typically smaller than the marker size. Systematic uncertainties are shown as boxes. Also shown are predictions based on the Lepto and Ariadne MC generators.
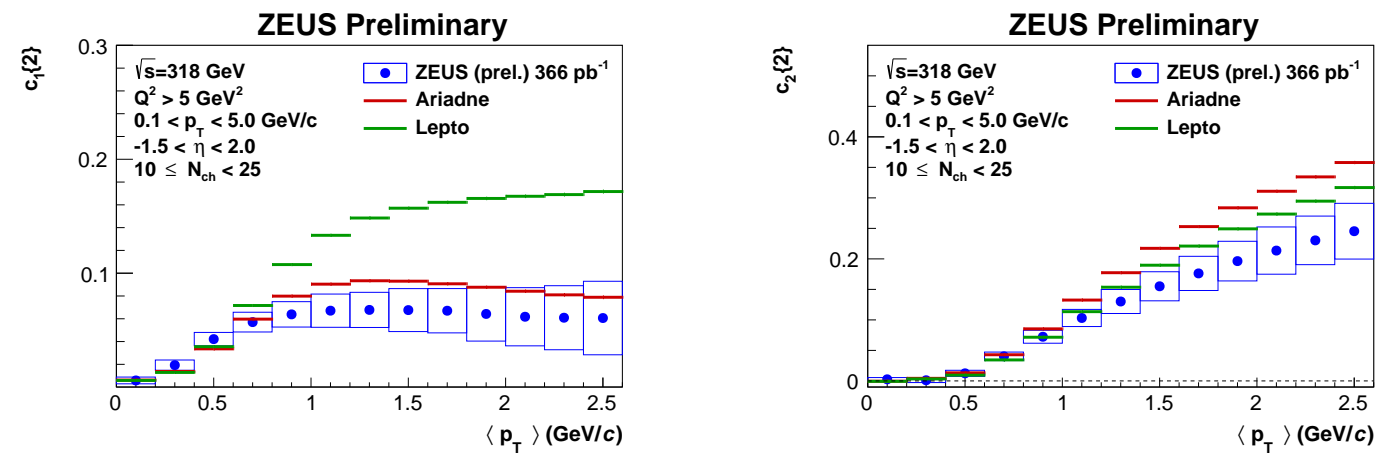

Figure 5: The correlation coefficient $c_{n}\{2\}, n=1,2$, as a function of $\left\langle p_{\mathrm{T}}\right\rangle$. The statistical uncertainties are shown as vertical lines although they are typically smaller than the marker size. Systematic uncertainties are shown as boxes. Also shown are predictions based on the Lepto and Ariadne MC generators. 


\section{References}

[1] ZEUS Collaboration, U. Holm (editor), The ZEUS Detector. Status Report, DESY (1993), URL http://www-zeus.desy.de/bluebook/bluebook.html.

[2] H. Abramowicz et al. [ZEUS Collaboration], Phys. Rev. D87, 052014 (2013).

[3] H. Abramowicz et al. [ZEUS collaboration], Phys. Lett. B715, 88 (2012).

[4] G. Ingelman, A. Edin, and J. Rathsman. Comput. Phys. Commun 101, 108 (1997).

[5] Leif Lonnblad. Comput. Phys. Commun. 71, 15 (1992).

[6] Torbjorn Sjostrand. PYTHIA 5.7 and JETSET 7.4: Physics and manual (1994). 